\title{
Selective MIMO diversity with subcarrier-wise UE antenna identification/selection
}

\author{
Fumiyuki Adachi ${ }^{\text {a) }}$ and Amnart Boonkajay \\ Research Organization of Electrical Communication, Tohoku University, \\ 2-1-1 Katahira, Aoba-ku, Sendai, Miyagi 980-8577, Japan \\ a)adachi@ecei.tohoku.ac.jp
}

\begin{abstract}
Antenna diversity is a powerful means to improve the bit error rate (BER) performance in wireless communication systems. In this paper, we propose selective multi-input multi-output (MIMO) diversity with subcarrier-wise user equipment (UE) antenna identification/selection in a doubly-selective fading channel. Pilot-aided channel estimation (PACE) is used for identifying/selecting the best UE antenna prior to data transmission and decision-feedback channel estimation (DFCE) is used to update the channel state information (CSI). It is confirmed by computer simulation that the proposed selective MIMO diversity provides the improved BER performance compared to MISO/SIMO diversity in low and high mobility environments.
\end{abstract}

Keywords: MIMO diversity, antenna selection, channel estimation, decision-feedback, linear prediction

Classification: Wireless Communication Technologies

\section{References}

[1] J. K. Cavers, "Single-user and multiuser adaptive maximal ratio transmission for Rayleigh channels," IEEE Trans. Veh. Technol., vol. 49, no. 6, pp. 2043-2050, Nov. 2000. DOI:10.1109/25.901862

[2] F. Adachi, H. Tomeba, and K. Takeda, "Introduction of frequency-domain signal processing to broadband single-carrier transmissions in a wireless channel," IEICE Trans. Commun., vol. E92-B, no. 9, pp. 2789-2808, Sep. 2009. DOI: 10.1587/transcom.E92.B.2789

[3] F. Adachi, A. Boonkajay, Y. Seki, and T. Saito, "MIMO channel estimation for time-division duplex distributed antenna cooperative transmission," Proc. the 13th Int. Wireless Commun. and Mob. Comput. Conf. (IWCMC 2017), Valencia, Spain, June 2017. DOI:10.1109/IWCMC.2017.7986288

[4] D. Chu, "Polyphase codes with good periodic correlation properties," IEEE Trans. Inf. Theory, vol. 18, no. 4, pp. 531-532, July 1972. DOI:10.1109/TIT. 1972.1054840

[5] J.-J. van de Beek, O. Edfors, M. Sandell, S. K. Wilson, and P. O. Borjesson, "On channel estimation in OFDM systems," Proc. IEEE Veh. Technol. Conf. (VTC1995), Chicago, USA, Vol. 2, pp. 815-819, July 1995. DOI:10.1109/ VETEC.1995.504981

[6] K. Takeda and F. Adachi, "Frequency-domain MMSE channel estimation for frequency-domain equalization of DS-CDMA signals," IEICE Trans. Commun., 
vol. E90-B, no. 7, pp. 1746-1753, July 2007. DOI:10.1093/ietcom/e90-b. 7.1746

\section{Introduction}

Antenna diversity is a powerful means to improve the bit error rate (BER) performance in a poor channel condition. Due to the hardware complexity and cost issue, only a few antennas are available at a user equipment (UE). The maximal-ratio transmit diversity (MRTD) [1] and the minimum mean square error based combining diversity (MMSECD) [2] are typical examples for OFDM downlink and SC uplink, respectively. All the necessary signal processing for diversity transmission and reception can be implemented at the base station (BS) side only. Since only single receive/transmit UE antenna is allowed to use in MRTD/ MMSECD, the achievable diversity gain is limited.

In this paper, to increase the diversity gain by using multiple UE antennas, we propose a selective multi-input multi-output (MIMO) diversity with subcarrier-wise UE antenna identification/selection. Pilot-aided channel estimation (PACE) is used for identifying/selecting the best UE antenna and for computing MRTD/ MMSECD weights at the BS side prior to data transmission. However, in a high mobility environment, the channel state information (CSI) acquired by PACE becomes outdated during data transmission period. Therefore, in this paper, the decision-feedback channel estimation (DFCE) is introduced. It is confirmed by computer simulation that the selective MIMO diversity provides the improved BER performance compared to MISO/SIMO diversity in low and high mobility environments.

\section{Identification/selection of best UE antenna and computation of MRTD/MMSECD weights}

BS and UE are assumed to have $N_{B S}$ antennas and $N_{U E}$ antennas, respectively. The equal number $N_{c}$ of subcarriers is assumed for OFDM downlink and SC uplink. The time-division duplex (TDD), which can exploit the channel reciprocity in antenna selection and filter weight calculation, is assumed. The subframe considered in this paper consists of uplink pilot time slot (UpPTS) and downlink PTS (DwPTS), followed by 12 data time slots (DTSs) [3]. As illustrated in Fig. 1, UE transmits the uplink pilots simultaneously from $N_{U E}$ antennas during UpPTS period

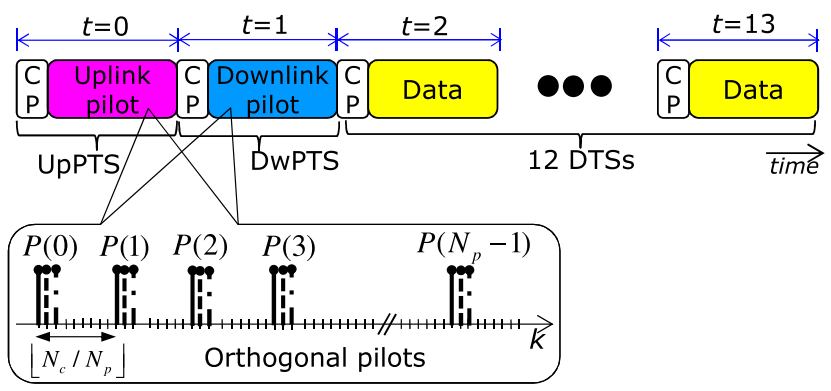

Fig. 1. Subframe structure. 
(slot time $t=0$ ). After performing PACE, BS selects the best UE antenna for each subcarrier and computes MRTD/MMSECD weights. Then, BS transmits the downlink pilots simultaneously from $N_{B S}$ antennas during DwPTS period (slot time $t=1$ ). The information about which UE antenna has been selected by BS is unknown to UE. Based on the CSI obtained by performing PACE, UE identifies the best UE antenna which has been selected by BS. Uplink and downlink pilots are a type of frequency-division multiplexed (FDM) pilot with equally spaced pilot subcarrier mapping, constructed by using the Zadoff-Chu sequence [4].

Throughout the paper, discrete-time representation normalized by inverse discrete Fourier transform (IDFT) sampling period $T_{s}=T / N_{c}$ where $T$ is the IDFT block length in time.

\subsection{Slot time $t=0$}

The uplink pilots transmitted simultaneously from $N_{U E}$ antennas are received by $N_{B S}$ antennas. Since each pilot is an FDM pilot with equally spaced pilot subcarrier mapping, the delay-time domain windowing technique [5] can be applied to obtain the channel gain estimates $\left\{\hat{H}_{m, n}(k, t=0) ; k=0 \sim N_{c}-1\right\}$, where $k$ denotes the subcarrier index.

BS identifies the best UE antenna for each subcarrier $k$ as

$$
\breve{n}(k)=\arg \max _{n=0 \sim N_{U E}-1} \sum_{m=0}^{N_{B S}-1}\left|\hat{H}_{m, n}(k, t=0)\right|^{2} .
$$

Hereafter, unless otherwise stated, index $k$ is omitted from $\breve{n}(k)$ for the sake of brevity. Assuming that the UE correctly selects the $\breve{n}$ th antenna, BS computes MRTD/MMSECD weights as

$$
\begin{gathered}
W_{m}^{M R T D}(k)=\frac{\left(\frac{\hat{H}_{m, \breve{n}}^{*}(k, t=0)}{\sum_{m=0}^{N_{B S}-1}\left|\hat{H}_{m, \breve{n}}(k, t=0)\right|^{2}}\right)}{\sqrt{\frac{1}{N_{c}} \sum_{k=0}^{N_{c}-1}\left(\sum_{m=0}^{N_{B S}-1}\left|\hat{H}_{m, \breve{n}}(k, t=0)\right|^{2}\right)^{-1}}}, \\
W_{m}^{M M S E C D}(k)=\frac{\hat{H}_{m, \breve{n}}^{*}(k, t=0)}{\sum_{m=0}^{N_{B S}-1}\left|\hat{H}_{m, \breve{n}}(k, t=0)\right|^{2}+\left(E_{S} / N_{0}\right)^{-1}},
\end{gathered}
$$

where $E_{s}$ represents the transmit data signal energy given by $E_{s}=S T_{s}$. The MRTD weight of Eq. (2) is a combination of the conventional MRTD weight [1] and 2-dimensional (antennas and subcarriers) power allocation for keeping the transmit power intact (i.e., always equal to $S$ ).

\subsection{Slot time $t=1$}

The downlink pilots transmitted simultaneously from $N_{B S}$ antennas are received by $N_{U E}$ antennas. Similar to the uplink PACE done at BS at $t=0$, UE obtains the channel gain estimates $\left\{\hat{H}_{m, n}(k, t=1) ; k=0 \sim N_{c}-1\right\}$ and identifies the $\breve{n}(k)$ th 
antenna according to Eq. (1) but by replacing $\hat{H}_{m, n}(k, t=0)$ with $\hat{H}_{m, n}(k, t=1)$. Since the uplink and downlink channels are reciprocal due to TDD and fading remains almost unchanged between $t=0$ and 1, the false identification of UE antenna is sufficiently small (this was confirmed by BER performance evaluation by computer simulation).

\section{Selective MIMO diversity}

\subsection{OFDM downlink}

BS multiplies its transmitting $N_{c}$-symbol data block $\left\{d(k, t) ; k=0 \sim N_{c}-1\right\}$ by the MRTD weight $\left\{W_{m}^{M R T D}(k) ; k=0 \sim N_{c}-1\right\}$ to get $\left\{\sqrt{2 S} W_{m}^{M R T D}(k) d(k, t) ; k=\right.$ $\left.0 \sim N_{c}-1\right\}$, which is to be transmitted from the $m\left(=0 \sim N_{B S}-1\right)$ th antenna. After $N_{c}$-point IDFT, the resultant time-domain signals are transmitted simultaneously from $N_{B S}$ antennas. UE transforms signals received on its $N_{U E}$ antennas by $N_{c}$-point DFT into the frequency-domain signals and then, selects the subcarrier component $R_{\breve{n}}(k, t)$ received on the $\breve{n}(k)$ th antenna. The antenna selection output $\left\{R(k, t) ; k=0 \sim N_{c}-1\right\}$ can be written as

$$
R(k, t)=\sqrt{2 S} H_{e}(k, t) d(k, t)+\Pi_{\breve{n}}(k, t),
$$

where $H_{e}(k, t)$ and $\Pi_{\breve{n}}(k, t)$ are the equivalent channel gain and the noise due to AWGN, respectively, and $E\left[|d(k, t)|^{2}\right]=1 . H_{e}(k, t)$ is given as

$$
\begin{aligned}
& H_{e}(k, t)=\sum_{m=0}^{N_{B S}-1} W_{m}^{M R T D}(k) H_{m, \breve{n}}(k, t) \\
& =\frac{\left(\frac{\sum_{m=0}^{N_{B S}-1} \hat{H}_{m, \breve{n}}^{*}(k, t=0) H_{m, \breve{n}}(k, t)}{\left.\sum_{m=0}^{N_{B S}-1}\left|\hat{H}_{m, \breve{n}}(k, t=0)\right|^{2}\right)}\right.}{\sqrt{\frac{1}{N_{c}} \sum_{k=0}^{N_{c}-1}\left(\sum_{m=0}^{N_{B S}-1}\left|\hat{H}_{m, \breve{n}}(k, t=0)\right|^{2}\right)^{-1}}}
\end{aligned}
$$

The symbol decision is made as

$$
\hat{d}(k, t)=\min _{d \in \mathbf{D}}\left|R(k, t)-\sqrt{2 S} \hat{H}_{e}(k, t) d\right|, \quad k=0 \sim N_{c}-1,
$$

where $d$ and $\mathbf{D}$ denote the symbol candidate and a set of candidate symbols, respectively. Since $H_{e}(k, t)$ is unknown to UE, it has been replaced with its estimate $\hat{H}_{e}(k, t) . \hat{H}_{e}(k, t=2)$ to be used for symbol decision at $t=2$ can be computed by replacing $\hat{H}_{m, \breve{n}}(k, t=0)$ and $H_{m, \breve{n}}(k, t)$ with $\hat{H}_{m, \breve{n}}(k, t=1)$ in Eq. (5). However, in a high mobility environment, $\hat{H}_{e}(k, t=2)$ deviates from the actual equivalent channel gain. Therefore, $\hat{H}_{e}(k, t)$ at $t=3 \sim 13$ is updated by using DF as

$$
\hat{H}_{e}(k, t)=\tilde{H}_{e}(k, t-1),
$$

where $\tilde{H}_{e}(k, t)$ is the equivalent channel gain estimate obtained by moving-average over $Q$ subcarriers to remove the perturbation due to symbol errors and noise as

$$
\tilde{H}_{e}(k, t-1)=\frac{1}{Q} \sum_{q=-(Q-1) / 2}^{(Q-1) / 2} \frac{R_{\breve{n}}(k+q, t-1)}{\hat{d}(k+q, t-1)} .
$$




\subsection{SC uplink}

UE transforms its transmitting $N_{c}$-symbol data block $\left\{d\left(t^{\prime}, t\right) ; t^{\prime}=0 \sim N_{c}-1\right\}$ into the frequency-domain signal $\left\{D(k, t) ; k=0 \sim N_{c}-1\right\}$ by $N_{c}$-point DFT and then, maps them to $N_{U E}$ antennas according to the subcarrier-wise antenna selection $\left\{\breve{n}(k) ; k=0 \sim N_{c}-1\right\}$. The resultant frequency-domain signal $\left\{\sqrt{2 S} D_{n}(k, t) ; k=\right.$ $\left.0 \sim N_{c}-1\right\}$ to be transmitted from the $n\left(=0 \sim N_{U E}-1\right)$ th UE antenna is transformed to the time-domain SC signal by $N_{c}$-point IDFT, where $D_{n}(k, t)=D(k, t)$ if $n=\breve{n}(k)$ and $D_{n}(k, t)=0$ otherwise. The resultant time-domain signals are transmitted simultaneously from $N_{U E}$ antennas.

After transforming the signal received on its $m$ th antenna by $N_{c}$-point DFT into the frequency-domain signal $\left\{R_{m}(k, t) ; k=0 \sim N_{c}-1\right\}$, BS performs MMSECD as

$$
R(k, t)=\sum_{m=0}^{N_{B S}-1} W_{m}^{M M S E C D}(k) R_{m}(k, t)
$$

which can be written as

$$
R(k, t)=\sqrt{2 S} H_{e}(k, t) D(k, t)+\sum_{m=0}^{N_{B S}-1} W_{m}^{M M S E C D}(k) \Pi_{m}(k, t),
$$

where $H_{e}(k, t)$ represents the equivalent channel gain and $\Pi_{m}(k, t)$ is the noise due to AWGN. $H_{e}(k, t)$ is given as

$$
H_{e}(k, t)=\frac{\sum_{m=0}^{N_{B S}-1} \hat{H}_{m, \breve{n}}^{*}(k, t=0) \hat{H}_{m, \breve{n}}(k, t)}{\sum_{m=0}^{N_{B S}-1}\left|\hat{H}_{m, \breve{n}}(k, t=0)\right|^{2}+\left(E_{S} / N_{0}\right)^{-1}} .
$$

After transforming $\left\{R(k, t) ; k=0 \sim N_{c}-1\right\}$ into the time-domain received signal $\left\{r\left(t^{\prime}, t\right) ; t^{\prime}=0 \sim N_{c}-1\right\}$ by $N_{c}$-point IDFT, the symbol decision is made as

$$
\hat{d}\left(t^{\prime}, t\right)=\min _{d \in \mathbf{D}}\left|r\left(t^{\prime}, t\right)-\sqrt{2 S}\left(\frac{1}{N_{c}} \sum_{k=0}^{N_{c}-1} \hat{H}_{e}(k, t)\right) d\right|,
$$

where $\hat{H}_{e}(k, t)$ is the estimate of $H_{e}(k, t) . \hat{H}_{e}(k, t=2)$ to be used for symbol decision at $t=2$ can be computed by replacing $H_{m, \breve{n}}(k, t)$ with $\hat{H}_{m, \breve{n}}(k, t=0)$ in Eq. (11). Similar to Eqs. (7) and (8), $\hat{H}_{m, \breve{n}}(k, t)$ is updated at $t=3 \sim 13$ using DF and MMSE based channel estimation (MMSECE) [6]. Then, $W_{m}^{M M S E C D}(k)$ is obtained by replacing $\hat{H}_{m, \breve{n}}(k, t=0)$ with $\hat{H}_{m, \breve{n}}(k, t)$ in Eq. (3) and $\hat{H}_{e}(k, t)$ is obtained by replacing both $\hat{H}_{m, \breve{n}}(k, t=0)$ and $H_{m, \breve{n}}(k, t)$ with $\hat{H}_{m, \breve{n}}(k, t)$ in Eq. (11). The time-domain MMSECD output $r\left(t^{\prime}, t\right)$ is computed by substituting $W_{m}^{M M S E C D}(k)$ into Eq. (9) and performing $N_{c}$-point IDFT. Then, by using $r\left(t^{\prime}, t\right)$ and $\hat{H}_{e}(k, t)$, symbol decision is made according to Eq. (12).

\section{Computer simulation}

The average uncoded BER performances of OFDM downlink and SC uplink with selective MIMO diversity are evaluated by computer simulation. It is assumed that BS and UE are equipped with $N_{B S}=4$ antennas and $N_{U E}=2$ antennas, respectively. $N_{B S} \times N_{U E}$ channels are characterized by independent and identically dis- 


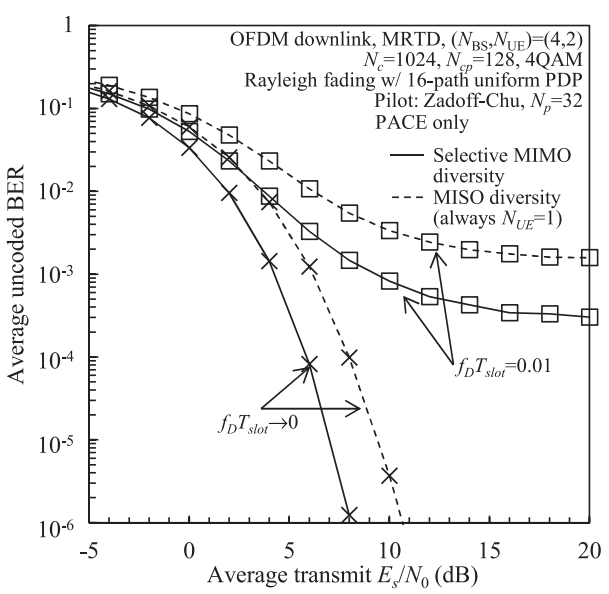

(a) OFDM downlink

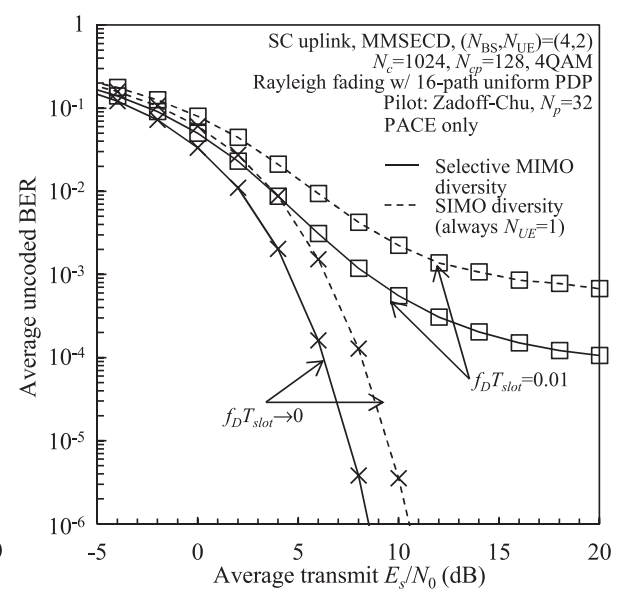

(b) SC upnink

Fig. 2. Average uncoded BER performance.

tributed doubly-selective block Raleigh fading channels having 16-path uniform power delay profile (PDP) with the maximum time delay $\tau_{\max }=15$. The number of subcarriers is $N_{c}=1024$ and the cyclic prefix (CP) length is $N_{c p}=128$. The Zadoff-Chu sequence [4] of $N_{p}=32$-symbol length is employed as the pilot symbol sequence $\left\{P(\omega) ; \omega=0 \sim N_{p}-1\right\}$. The perfect knowledge of transmit $E_{s} / N_{0}$ is assumed for MMSECD and MMSECE. 4QAM data modulation is assumed. We found that $Q=11$ is near the optimum to minimize the BER.

The simulated average uncoded BER performance with selective MIMO diversity using PACE only is plotted as a function of the average $E_{S} / N_{0}$ for the normalized maximum Doppler frequency $f_{D} T_{\text {slot }} \rightarrow 0$ and 0.01 in Fig. 2, where $f_{D}$ and $T_{\text {slot }}$ denote the Rayleigh fading maximum Doppler frequency and the time slot length equal to $\left(N_{c}+N_{c p}\right) T_{s}$, respectively. It can be confirmed that by allowing the use of 2 UE antennas, selective MIMO diversity provides the improved BER performance compared to MISO/SIMO diversity.

The required transmit $E_{S} / N_{0}$ for achieving the average $\mathrm{BER}=10^{-6}$ when $f_{D} T_{\text {slot }} \rightarrow 0$ (i.e., low mobility) can be reduced by about $2 \mathrm{~dB}$ and the BER floor observed when $f_{D} T_{\text {slot }}=0.01$ (i.e., relatively high mobility) is reduced by more than 5 times.

\section{Conclusion}

Since only single receive/transmit UE antenna is allowed to use in MRTD/ MMSECD, the achievable diversity gain is limited. In order to increase the diversity gain by using multiple UE antennas, this paper proposed the selective MIMO diversity with subcarrier-wise UE antenna selection. PACE is used for selecting the best UE antenna prior to data transmission and DFCE is used to update the CSI to track the time varying channels in a relatively high mobility environment. The computer simulation confirmed that the selective MIMO diversity provides the improved BER performance compared to MISO/SIMO diversity. 


\section{Acknowledgments}

The results presented in this paper have been achieved by "The research and development project for realization of the fifth-generation mobile communications system," commissioned to Tohoku University by The Ministry of Internal Affairs and Communications (MIC), Japan. 